\title{
SwissDRG 6.0: nécessité d'une stratégie actualisée pour l'avenir
}

\author{
Petra Ingenpass a , Beatrix Meyer ${ }^{b}$ \\ a $\mathrm{Dr}$ med., cheffe adjointe de la division Tarifs et économie de la santé pour les médecins hospitaliers \\ ${ }^{b}$ Cheffe de la division Tarifs et économie de la santé pour les médecins hospitaliers
}

La version 6.0 de SwissDRG a reçu à nouveau de nombreux changements basés sur des données d'une qualité toujours insuffisante. Le temps est venu de faire le point de la situation et d'actualiser la stratégie de développement.

\section{Définir la stratégie actuelle et fixer des mesures pour sa réalisation}

Le développement de SwissDRG se fonde sur la stratégie 2013+, élaborée par SwissDRG SA en 2012, en collaboration avec ses partenaires. Si certains objectifs stratégiques ont déjà été atteints, pour d'autres, peu de progrès sont visibles. La FMH estime par conséquent qu'il est temps de procéder à une analyse et à un état des lieux de l'évolution de SwissDRG. Cette analyse permettra de déterminer dans quelle direction le tarif devra se développer à l'avenir. La FMH pense qu'il est important que SwissDRG SA actualise sa stratégie et montre quelles sont les mesures à prendre pour la mettre en œuvre. Selon la stratégie 2013+ de SwissDRG SA, la structure tarifaire doit permettre d'expliquer les différences de coûts entre les fournisseurs de prestations, sur la base d'environ 1200 DRG, y compris les rémunérations supplémentaires. Mais on peut se demander dans quelle mesure cet objectif est compatible par exemple avec la poursuite de la différenciation de la structure tarifaire adoptée actuellement par le conseil d'administration de SwissDRG SA pour mieux prendre en compte les cas très coûteux et fortement déficitaires. En effet, il est probable que le nombre de forfaits par cas (DRG) augmente, comme les rétributions additionnelles qui sont aussi nécessaires, car elles permettent un remboursement plus adéquat. C'est un exemple parmi tant d'autres qui montre la pertinence d'un réexamen de la stratégie mise en place.

\section{Améliorer la structure tarifaire: le conseil d'administration donne une valeur indicative}

Selon SwissDRG SA, il faut admettre que les forfaits par cas n'expliquent pas toutes les différences de coûts entre les hôpitaux. Il existe en effet des différences régionales concernant les salaires et les charges sociales. base fixes pour les infrastructures hautement spécialisées ou les services d'urgence qui sont nécessaires sans être pleinement exploitées (par ex. services d'isolement particuliers). Mais aussi les cas fortement déficitaires génèrent des pertes lorsqu'ils ne sont pas compensés par des cas bénéficiaires. Ces différences de coûts, qui ne découlent pas directement de l'efficacité et de l'efficience des hôpitaux, peuvent être rééquilibrées lors des négociations tarifaires au moyen d'une différenciation des prix.

Après l'extraction correcte des coûts d'exploitation et des coûts d'utilisation des immobilisations relevant de l'AOS, le conseil d'administration de SwissDRG SA prévoit une marge indicative de $\pm 5 \%$ pour le calcul des coûts moyens par cas; cet objectif devant être atteint d'ici fin 2019. Pour la FMH, il est essentiel que SwissDRG SA inscrive dans sa stratégie quelles mesures permettent de l'atteindre ou, plus précisément, comment la structure tarifaire peut être améliorée sur ce point.

\section{Demande d'un rapport final détaillé pour plus de transparence et de clarté}

Lors de la présentation de SwissDRG version 6.0, SwissDRG SA a indiqué plusieurs nouveautés et transformations. Cependant, le degré de détails et la pertinence de ces informations sont parfois très différents. Ainsi, le regroupement de toutes les prestations concernant l'appendicite a été présenté de manière exhaustive alors que la tarification des DRG de réhabilitation précoce, non tarifés jusqu'ici, n’a pas été traitée en détails. Dans la version 6.0, plusieurs prestations ont été transférées dans la pré-MDC ${ }^{1}$, dans laquelle étaient pris en compte à l'origine les traitements coûteux, mais aussi pluridisciplinaires, tels que les transplantations ou les ventilations artificielles. Dès lors, les caractéristiques communes des DRG transférés des autres chapitres classés selon les organes dans la pré-MDC peuvent être celles d'un traitement complexe, de soins intermédiaires ou d'une réhabilitation précoce. Très peu d'explications sont fournies sur la manière de procéder. Si on évite ainsi des fractionnements de DRG ou l'utilisation de DRG d'erreur, on accorde aussi toujours plus d'importance aux procédures au détriment des diagnostics. La FMH estime qu'il est nécessaire que SwissDRG SA 
présente les développements et les modifications de la structure tarifaire de manière plus transparente et claire dans un rapport final complet et détaillé.

\section{Amélioration des données: toujours d'actualité}

SwissDRG SA a déjà pris plusieurs mesures pour améliorer la qualité des données, par exemple, avec l'obligation de saisir les coûts par cas selon le système REKOLE ${ }^{\circledR}$. De plus, elle a intensifié ses échanges avec les hôpitaux pour le contrôle de la plausibilité des données et leur offre désormais la possibilité de l'effectuer en cours d'année sur un portail des données. Selon SwissDRG SA, un net besoin d'amélioration demeure, tant pour la saisie des données de coûts que pour le codage des prestations fournies. En effet, pour le développement de SwissDRG 6.0, il a fallu supprimer l'ensemble des données de 16 hôpitaux. Pour le moment, SwissDRG SA ne peut pas évaluer la qualité des données de coûts d'utilisation des immobilisations $(\mathrm{CUI})^{2}$. Mais celle-ci ne devrait guère s'être améliorée; l'écart de la part moyenne des CUI aux frais d'exploitation entre les hôpitaux reste en effet très élevé (entre env. 4\% et 18\%). En 2015, SwissDRG SA a décidé que, dès l'année de données 2016, les hôpitaux devaient fournir les CUI exclusivement selon le système REKOLE ${ }^{\circledR}$ pour la suite du développement de SwissDRG. Cette mesure est certes importante, mais on peut douter qu'elle soit suffisante pour obtenir la qualité requise de ces données.

L'exigence d'une amélioration de la qualité des données figurait déjà dans la stratégie de développement 2013+. Comme les mesures prises jusqu'ici n'ont pas suffi pour obtenir une qualité adéquate des données, il faut en essayer d'autres. En même temps, les objectifs stratégiques doivent définir la manière de procéder au cas où une amélioration durable de la qualité des données ne devait pas voir le jour.

\section{Prendre rapidement en main le problème des cas fortement déficitaires}

SwissDRG SA a décidé de rechercher des solutions pour la rémunération des cas très coûteux et fortement déficitaires au sein de la structure tarifaire, en tenant compte des cas bénéficiaires. A cet effet, il s'agit de tester des méthodes de calcul, mais aussi de considérer des rétributions additionnelles pour des constellations déterminées de cas fortement déficitaires. De tels cas sont notamment plus fréquents lors de séjours de longue durée, de transfert en provenance d'un autre hôpital ou de traitement en soins intensifs.

SwissDRG SA présente des évaluations concernant les cas coûteux et fortement déficitaires dans la version
6.0 de SwissDRG. Elle y définit qu'un cas est fortement déficitaire à partir d'un coût global de 400000 francs et d'un déficit de 40000 francs. Dans la version 6.0, 76 cas de 14 hôpitaux correspondent à cette définition et génèrent un déficit global de 12 millions de francs. Pour la version 5.0, il y avait 56 cas de 12 hôpitaux avec un déficit global de 11 millions de francs ${ }^{3}$. Pour la FMH, l'inclusion des coûts globaux dans la définition des cas fortement déficitaires n'est pas pertinente; seul le montant du déficit importe.

Afin de pouvoir mieux prendre en compte les cas de traitement fortement déficitaires, il faut tenter toutes les approches possibles dans le cadre donné. SwissDRG SA a par exemple de nouveau essayé les suppléments pour high-outliers. Pour 347 forfaits par cas (DRG), le supplément pour high-outliers propre au DRG a été adapté. Mais il s'agit également de vérifier si des développements correspondants du système allemand G-DRG sont compatibles, par exemple, avec une extension des niveaux de complication et de comorbidité.

\section{Tous les DRG de réhabilitation précoce évalués malgré une base de données peu robuste}

Dans la version 6.0, tous les DRG de réhabilitation précoce sont pour la première fois évalués. La demande du Conseil fédéral visant à éviter si possible les forfaits par cas non évalués a ainsi été satisfaite. Les cas de réhabilitation précoce neurologique-neurochirurgicale et pluridisciplinaires sont attribués soit à deux DRG de la pré-MDC indépendants du diagnostic principal, soit à deux DRG de neurologie. Alors que la réhabilitation précoce neurologique et neurochirurgicale est clairement définie, l'établissement de caractéristiques minimales pour la réhabilitation précoce pluridisciplinaire fait défaut. Le code doit urgemment être défini en tenant compte des sociétés médicales concernées.

\section{Conclusions de la FMH}

Le moment est venu de faire le point de la situation. Selon la FMH, il est important que SwissDRG SA actualise sa stratégie et montre quelles mesures permettront de mettre progressivement en œuvre les objectifs stratégiques. Il s'agit en particulier de présenter comment la structure tarifaire peut être améliorée et comment la marge indicative de $\pm 5 \%$ pour le calcul des coûts moyens, telle que l'a adoptée le conseil d'administration, peut être atteinte d'ici à fin 2019. Et enfin, il faut apporter davantage de transparence dans les développements de la structure tarifaire. Les années à venir nous réservent encore un certain nombre de défis à relever. 